\section{P295 EVALUATION OF FRENCH MICROBIOLOGY LABORATORIES PRACTICES CONCERNING ANTIBIOTIC THERAPY AND ANTIMICROBIAL RESISTANCE}

${ }^{1,2} \mathrm{~A}$ Durocher, ${ }^{3} \mathrm{~V}$ Vernet-Garnier, ${ }^{2} \mathrm{M}$ Cornille, ${ }^{2} \mathrm{M}$ Delzenne, ${ }^{2} \mathrm{M}$ Methenni, ${ }^{2} \mathrm{D}$ Rousseau, ${ }^{2} \mathrm{E}$ Van Hecke. ${ }^{1}$ Haute Autorité de Santé (HAS), Saint Denis, France; ${ }^{2}$ ILIS- Lille 2 University, Lille, France; ${ }^{3} \mathrm{CHU}$ - Reims University, Reims, France

\section{0:1136/bmjqs-2013-002293.244}

Background Guidelines on prevention of bacterial resistance and quality of microbiology labs have been promoted by HAS.

Objectives Analysis of implementation of guidelines in 2011 versus 2009.

Methods Checklists for practices of 230 representative hospital labs.

Results Between 2009 and 2011, there was an increase in (i) survey of bacterial resistance by labs ( $98 \%$ vs $95 \%$ ), (ii) internal quality control $(88 \%$ vs $85 \%$ of public, $71 \%$ vs $64 \%$ of private labs $-\mathrm{p}=0.01)$, (iii) evaluation of procedures $(67 \%$ vs $57 \%-\mathrm{p}=0.01$ ), (iv) basic service maintained in $89 \%$ vs $85 \%$ of labs, (v) information system (96\% vs $88 \%$ of public, $82 \%$ vs $75 \%$ of private labs $-\mathrm{p}=0.003$ ), (vi) liaison with the pharmacy ( $86 \%$ vs $78 \%$ of public, $76 \%$ vs $63 \%$ of private labs), (vii) epidemiological surveillance ( $96 \%$ vs $87 \%$ ), (viii) alert system $(97 \%$ vs $93 \%)$ mostly by computer in public or by phone in private labs). Results on resistance rates were more often submitted to the committee for the prevention of hospital infection ( $97 \%$ vs $93 \%)$ or of anti-infectives ( $77 \%$ vs $71 \%)$, to the clinical departments in $62 \%$ vs $50 \%$ of public, $85 \%$ vs $75 \%$ of private hospitals $(\mathrm{p}=0.018)$. Bacterial resistances were more often compared with antibiotic consumption in $57 \%$ vs $45 \%$ of general, $86 \%$ vs $61 \%$ of university, $37 \%$ vs $34 \%$ of private hospitals $(\mathrm{p}=0.002)$

Conclusions Quality of microbiology laboratory practices increased since 2009 but efforts have to be continued in evaluation of internal quality procedures, communication about bacterial resistances to clinical departments and comparison resistances-antibiotic consumption.

\section{P300 THEORY-INFORMED TAILORED IMPLEMENTATION OF GUIDELINES IN SWEDISH PRIMARY CARE PHYSIOTHERAPY: DEVELOPMENT OF A STRATEGY}

${ }^{1,2} \mathrm{~S}$ Bernhardsson, ${ }^{2} \mathrm{~K}$ Johansson, ${ }^{3} \mathrm{P}$ Nilsen, ${ }^{2} \mathrm{~B}$ Öberg, ${ }^{1,4} \mathrm{M}$ Larsson. ${ }^{1}$ Region Västra Götaland, Primary Care, Gothenburg, Sweden; ${ }^{2}$ Linköping University, Department of Medical \& Health Sciences, Div of Physiotherapy, Linköping, Sweden; ${ }^{3}$ Linköping University, Department of Medical \& Health Sciences, Div of Healthcare, Linköping, Sweden; ${ }^{4}$ Sahlgrenska Academy at Gothenburg University, Inst of Neuroscience \& Physiology, Gothenburg, Sweden

\section{0:1136/bmjqs-2013-002293.245}

Background There is little knowledge about effective guideline implementation strategies in primary care physiotherapy. The use of theory for the development of implementation strategies is advocated by some, a pragmatic approach by others.

Objectives To adapt a theoretical model to Swedish primary care physiotherapy using a pragmatic approach, to identify barriers and facilitators for the use of guidelines in physiotherapy, and to tailor an intervention to address these.

Methods We searched the literature for evidence of effectiveness of implementation strategies. We analysed various implementation theories and selected the Grol 5-step model to guide our implementation process. We adapted the model to the context in which we wanted to introduce the guidelines. We identified barriers and facilitators and possible intervention components to address these via a survey and a literature review.

Results The adaptation and addressing the identified barriers and facilitators resulted in a tailored, evidence-based, theoryinformed implementation strategy. The most important barriers identified were lack of time, low awareness, knowledge, and access to guidelines. An active, multifaceted implementation intervention was developed. The main implementation activity was a seminar with group discussions. Other components were a website, email reminders, email support, and patient information leaflets.

Discussion Using a model to systematically develop a strategy and a pragmatic approach to adapt it to the context in which it was to be implemented, helped to identify barriers and facilitators and to tailor an implementation intervention to Swedish primary care physiotherapy.

Implications for Guideline Developers/Implementers A theoryinformed but pragmatic approach can be recommended.

\section{P303 SUCCESSFUL IMPLEMENTATION OF INFECTION PREVENTION AND MANAGEMENT GUIDELINES IN AN INTEGRATED HEALTH CARE SYSTEM: STRUCTURE, PROCESS AND OUTCOMES}

J Harris. The Permanente Medical Group, San Rafael, USA

\section{0:1136/bmjqs-2013-002293.246}

Background Infection is one of the leading causes of inpatient morbidity and mortality. Sepsis, central line infections, surgical site infections, ventilator-acquired pneumonia and c. difficile infections account for the majority of these cases.

Objectives To describe the process, structure and results of efforts to better manage sepsis, central line infections, surgical site infections, and hospital acquired infections in an integrated health system using organisational best practices; to present results of these risk reduction and proactive disease treatment programmes

Methods Analysis of morbidity and mortality was the first step. Evidence for effective practices was assessed. Pilot projects based on the best evidence were conducted and results disseminated by organisation leaders, who emphasised doing the right thing at the right time all the time. Suggested order sets were incorporated in the EMR. Progress towards goals was disseminated regularly.

Results Mortality rates for severe sepsis dropped substantially over the last 4 years. Inpatient clostridium difficile infections, blood stream infections, and surgical site infections have also dropped dramatically.

Discussion Guideline recommendations were adopted across our delivery system when supported by top leadership, testing, training, specific care processes, EMR prompts for tests and treatments, regularly reviewed process metrics and group financial incentives. Practice variance was reduced and outcomes markedly improved.

Implications for Guideline Developers Guideline recommendations are more likely to be adopted in a uniform manner if they include specific recommendation, suggestions for implementation use in organised settings, and process and outcome metrics to track improvements. 